\title{
Cloud-Based Spatial Information Service Architecture within LBS
}

\author{
Tingyan Xing1, Shang Zhang1,2, Liufeng Tao ${ }^{1}$ \\ ${ }^{1}$ Faculty of Information Engineering, China University of Geosciences, Beijing, China \\ ${ }^{2}$ College of Computer and Information Technology, China Three Gorges University, Yichang, China \\ Email: xtygis@163.com, wetoo@163.com
}

Received 27 March 2014; revised 27 April 2014; accepted 4 May 2014

Copyright (C) 2014 by authors and Scientific Research Publishing Inc.

This work is licensed under the Creative Commons Attribution International License (CC BY).

http://creativecommons.org/licenses/by/4.0/

(c) (i)

\begin{abstract}
Location Based Services (LBS) have become a popular technology to retrieve information about the surroundings of a mobile user which results in ubiquitous demand of spatial information service with diverse needs of different types of users. The aim of this paper is to reveal the potential of cloud-based spatial information service architecture that plays an integral role in LBS design and practice. This paper analyzes the characteristics of the spatial information of cloud services, such as data access transparency, spatial analysis parallelization, service capabilities flexible, information services standardization, service aggregation visualization, re-development flexible. This paper provides a possible solution to overcome the LBS service issues. In this paper, the short review of LBS and Cloud computing are given first and then the possibility of LBS design with cloud computing are analyzed. At last, cloud-based spatial information service architecture is proposed.
\end{abstract}

\section{Keywords}

Cloud Computing, Spatial Information Cloud Services, LBS, Introduction

\section{Introduction}

Spatial Information technology prompted the generations of Location Based Services (LBS), a new spatial information era [1]. The target of this new era of spatial information includes not only the government, research institutions, businesses and other professional users, but also the public. How to solve the massive integration of heterogeneous data storage and management, processing, personalized service on demand and other technical problems, has driven scientists to focus on meeting the diverse needs of different types of users, providing the ubiquitous demand of location-based information service, which is considered as one of the most promising ap- 
plications of GIS.

Cloud computing is a shared infrastructure, which allows the application to run on a massive network connected computer system, which has a lot of features, such as scalable computing, centralized management, open function, massive storage and lightweight client [2].

At same time, mobile carriers providing location services are using different network interfaces, protocols and standards, and flexible strategy is an adequate mean to push current developments within the GIS and Spatial process domain, especially for the ongoing research and development topic "cloud". The availability of Cloud computing for Geographic Information systems (GIS) has increased tremendously in recent years [3], the service agent can easily and efficiently deploy onto the cloud infrastructure, through the most widely used web-based service.

\section{Brief Review of LBS}

\subsection{LBS}

With the rapid advances in wireless telecommunication and portable devices technologies, Location Based Services (LBSs) provide personalized services to the mobile subscribers based on their current position using GNSS (Global Navigation Satellite System), GIS (Geographic Information System) and WSN (Wireless Sensor network) technologies, and the tool for efficient management and continuous control. More and more people involve LBS in their industry and day-to-day life to better achieve their goals [4].

Several usage trends have been observed in location based services among emergency services, information services, tracking services and entertainment services where the most general [4].

Traditional LBS mainly uses the GPS positioning method, which can't offer services in indoor environment, in which most of human activities took place. LBS suited for outdoor and indoor environment and fused various positioning method, is the key to further development of LBS applications.

This paper focused on the research of LBS model framework, which is under outdoor and indoor environment and mixed of multiple location mode, carefully analyzed most of common LBS application services and summarized their commons.

\subsection{Brief State of the Art of Cloud}

Cloud computing is a commercial computing model, and it will distribute the task in a large pool of resources which are constituted by computer networks, enabling user's on-demand access to computing power, such as storage space and information services.

One of most important concept behind cloud computing is scalability, the key of which is virtualization, this key gathers multiple operating systems and applications on a Shared computer, in order to use the server more efficient.

Cloud computing has become a popular technology to provide spatial information, which includes transparent data accessing, parallel spatial analysis, flexible service, standard information services.

The essential technologies of Cloud computing include distributed spatial data storage strategy, virtual allocation model of compute nodes task, tile-based dynamic map release and parallel databases and Map Reduce processing model [5].

To achieve the optimal allocation of applications and computing resources, the kernel is the spatial and temporal characteristics of the spatial data.

Through cloud computing, geographic information systems core functions, such as dynamic projection and spatial analysis of multidimensional data, multi-coordinate system of automatic data collection can be integrated into the cloud computing system.

Cloud computing that can be thought includes the following several levels of service: infrastructure as a service (IaaS), platform as a service (PaaS) and software as a service (SaaS).

\section{Features of Spatial Cloud Computing}

As mentioned above, spatial information cloud service includes: data access transparency, spatial analysis parallelization, flexible and standard service, service visual aggregation, flexible secondary development. 


\subsection{Data Access Transparency}

Spatial information services relate to the various types of data with different data formats, in order to reduce the difficulty of user accessing, spatial information service platform based on cloud computing must support a variety of heterogeneous spatial data sources, and provide standard spatial data services to users with access method transparent. Consistent with the system configuration of space data source, the user could freely access the spatial data from different data sources [6].

\subsection{Parallel Spatial Analysis}

The data processing of spatial information services are massive, multi-source, heterogeneous, which totally are intensive tasks. But the power of single CPU computing is limited. Usually those kinds of tasks need to take full advantage of cloud computing on multi-CPU platform. At this point, parallel algorithms and strategy which could improve the processing efficiency is essential [6].

\subsection{Flexible Service}

Cloud computing technology has elastically expanded computing power, storage space and resource which would be dynamically allocated and shared through networks. Based on cloud computing, spatial information services are capable and scalable. First, this technology provides the different services and scalable storage capacity to multiple users, with the different needs; second, to the same service, the strength of its services (such as complex calculation of space services and space scalability for the huge amount of data) are extensible [6].

\subsection{Information Services Standardization}

Through the spatial information service platform, Cloud computing provided a variety of IT resources as a service to the user. Spatial data and processing capacity could be delivered via the internet to the user as service. To make no different access method to user, Service Standardization is the premise of sharing and interoperability.

\subsection{Visualized Service Aggregation}

Spatial information services include the types and functions of different service resources. In order to reuse heterogeneous resources, spatial cloud services platform with heterogeneous resources aggregation and visualization should achieve the efficient service, resource reuse and service flexibility.

\subsection{Secondary Development Flexible}

Different users, spatial information services, data management, spatial analysis and visualization are always different. To improve the availability of spatial information services platform, the platform must provide the flexible software reengineering environment, user-friendly interface and fast develop tools.

\section{Spatial Information Cloud Services Architecture}

The cloud-based spatial information services framework mainly includes five parts, infrastructure layer, resource pool layer, service management layer, user's portal and safety management module. The system architecture is shown in Figure 1.

\subsection{Infrastructure Layer}

The infrastructure is implemented as the basis of the entire spatial information cloud services, which are composed of a certain scale IT infrastructure, including servers, storage, networking, and load balancing.

\subsection{Resource Pool Layer}

Resource pool layer is based on the infrastructure layer, using a variety of techniques such as virtualization, reasonable granularity of resource package and automate way to manage and maintain in the background to ensure that the resources provided in time according to changes of user demand and dynamic allocation to meet the high 


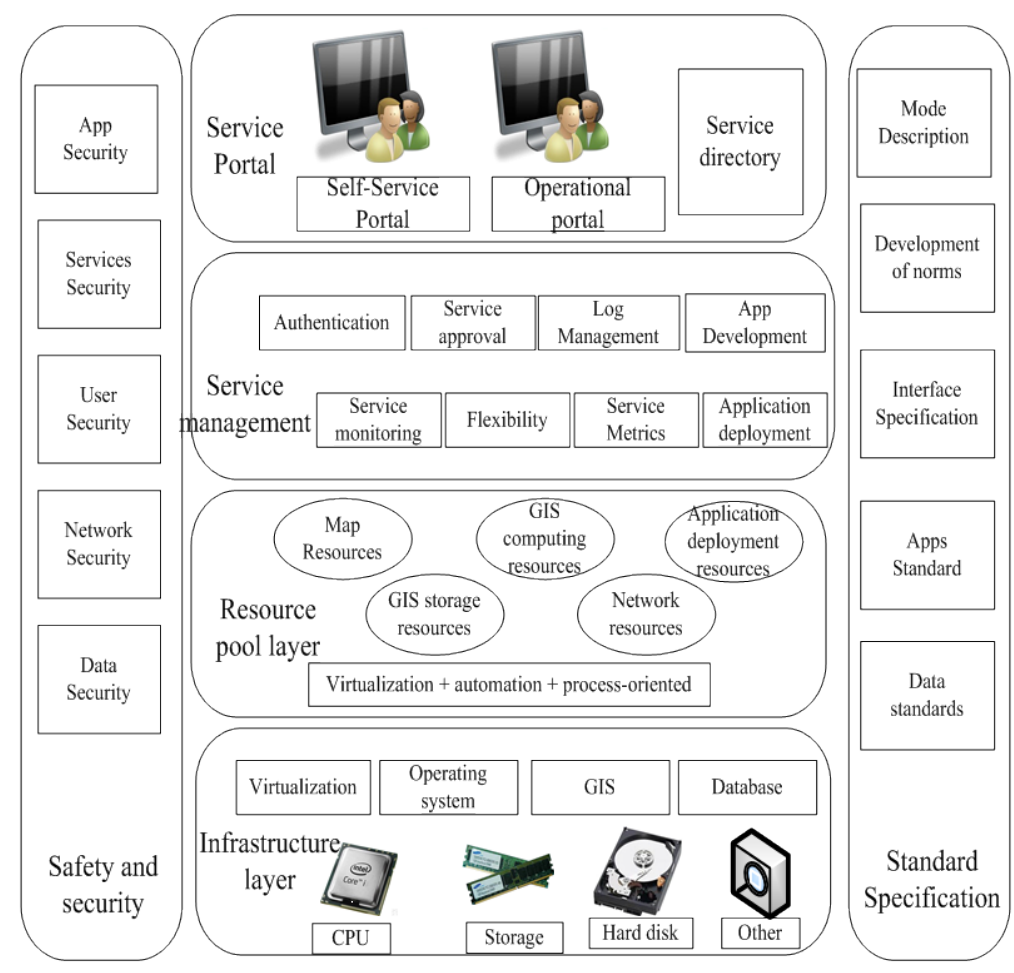

Figure 1. System architecture.

availability and high scalability of the application [7].

\subsection{Service Management Layer}

Service management is primarily responsible for managing user services, and monitoring how services using a variety of resources, and to achieve flexibility to allocate resources. Flexible allocation includes two aspects: first, according to the feedback of control information through the service management module to achieve the flexibility to adapt resources to meet the user's requirements; On the other hand, the management of the service can achieve dynamic resource allocation, without participation management.

\subsection{User Portal}

Portable service always reduces the cost of services, and is an important way to improve service convenience and user's experience. Through portable service, the user can demand the resources needed; the whole process is like ordering goods on e-commerce sites as easy.

\subsection{Safety Management Module}

Safety management module is to ensure that safety is an important guarantee for the entire services environment, not only to encrypt data in the process of storage, transmission, but also access to the user's authentication and access control, which ensures that different users have the respective safe working space.

\section{A Protype Spatial Service Platform Design}

Data is the foundation of all applications and services. The uniqueness and accuracy are the important characteristics of a geographic entity; therefore, in this spatial service platform differences and ambiguity caused by the multiple data sources must be overcome [8]. This platform (as shown in Figure 2) in the spatial service based service is to deal with all kinds of basic data, including the navigation electronic maps of all kinds of manufacturers, all kinds of industry data and remote sensing image data. Spatial service based service mainly has two parts, one is an online service support system, another is a middle-ware system. The design, implementation, 
operation of these two parts require rigorous data standard and constraint service specification.

\subsection{Online Support Service System}

Online service support system, based on the data management module conducts the most basic data management, multi-source data fusion, data processing and distribution, the diversity of data services, third-party data service support and so on. The design is to build a quick compatibility and integration of a variety of data sources of standard geographic data management system.

In establishing such a unified geographic data management system, flexible scheduling and data dispatch module are needed. Due to the diversity of the spatial service and the growing demand for the industry, different service requirements for data format are also diverse. Through data scheduling and dispatch module, and according to the data content choice, the user can specify its own data format needs, and carry on the content data release, data integration and updating. Therefore, all data are standardly unified and constant. The diversification is also the important guarantee of accurate spatial service. According to industry application and characteristics of the public service, while through the data access middle-ware and services middle-ware interface, the spatial service platform can be customized [9].

The Nav service support module is a platform to support various types of online services, and it mainly includes various navigation electronic map service functions, such as spatial analysis, buffer analysis, data scheduling, information processing, online service application, user management, and other functions. Those functions are designed to provide online function module for the user of third party, and also for the middle-ware design and development, which can be composed for all kinds of navigation application.

\subsection{The Middle-Ware Platform}

Middle-ware system is the core of spatial service, which throughout the underlying data management to online

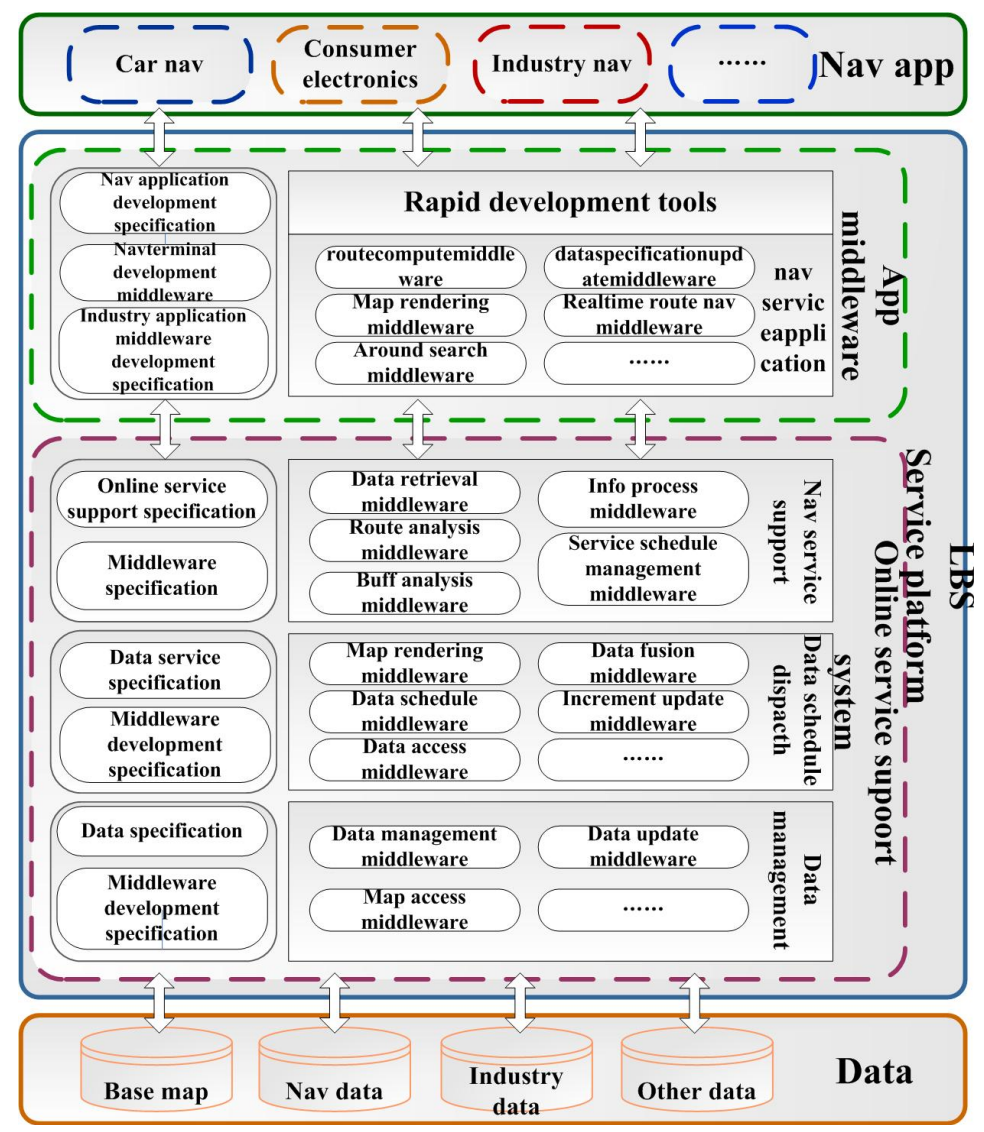

Figure 2. The architecture of spatial service platform. 
services support, and the application development and middle-ware technology process.

The service platform includes various types of middle-ware. In order to achieve structure flexible and functional independence, function modules are based on middle-ware implementation, which are basement of the infrastructure of the services platform.

In the field of electronic map navigation application, middle-ware is the main form and content of navigation terminal development, their complementation are completely followed by the specification shown in the Figure 1. In development, the use of middle-ware can have better classification of all kinds of functions and extensions, which can assist the user to build and deploy the spatial services.

Otherwise the rapid development tools are, moreover, providing an important feature of spatial service platform, which increase the difficulty of software development, shorten the development cycle, and solve the problem of multiplication differences. The tool can support multiple mobile devices in terms of building type development; the development of the core functionality can be implemented directly by the middle-ware in the form of drag and drop. Rapid development tools make full use of the navigation terminal development, middle-ware and industry application which could improve the efficiency of software development, enhance its extensibility [10].

\section{Conclusions}

By use of existing cloud computing technology, LBS spatial public service will also change the ordinary way of life, bringing enormous business opportunities.

The idea of the application of cloud computing, the establishment of geospatial data-based application services, cloud computing environment-SaaS philosophy will greatly increase the range of applications of spatial information and application level.

At present, the high cost of service influences the spatial public service. The development and application of Cloud based product will provide spatial service with a high efficiency and low cost.

Cloud computing can absorb scientific and technological achievements in the field of information technology synchronously and keep pace with the most advanced concept of software architecture, so as to promote the development of spatial service technology and software.

\section{Acknowledgments}

At the point of finishing this paper, I'd like to express my sincere thanks to all those who have lent me hands in the course of my writing this paper. First of all, I'd like to take this opportunity to show my sincere gratitude to my supervisor, Prof. Xincai Wu, who has given me so much useful advices on my writing. Secondly, I'd like to express my gratitude to my classmates who offered me references and information on time. Last but not the least, I'd like to thank those leaders, teachers and working staff especially those in the China University of Geosciences.

This paper is funded by the National Science \& Technology Pillar Program during the 12th Five-year Plan Period of China (2011BAH06B04; 2012BAB11B0501).

\section{References}

[1] Li, D.R. and Shao, Z.F. (2001) The Discuss of New Era of Geographic Information. Science in China, 39, 579-587.

[2] Liu, Y., Guo, W. and Jiang, W.T. (2009) Research of Remote Sensing Service Based on Cloud Computing Mode. Application Research of Computers, 26, 3428-3431.

[3] Sabbour, A.A. (2007) WiGuide: Indoor System for LBS. German University in Cairo.

[4] Sadoun, B. and Al-Bayari, O. (2007) LBS and GIS Technology Combination and Applications. Proceedings of IEEE/ACS International Conference on Computer Systems and Applications (AICCSA 07), 13-16 May 2007, Amman, Jordan, 578-583.

[5] Wu, L. and Zhang, Y. (2002) The Integrated Framework on Distributed Multispatial Database System. Geography and Territorial Research, 18, 6-10.

[6] Zhou, S.P., Hu, M.S. and Fang, F. (2010) The Concept Model and Technologies of Seamless Spatial Data Center. Earth Science-Journal of China University of Geosciences, 35, 345-350.

[7] Wu, X.C. (2009) Data Center Integration Development Technology: The Next Generation GIS Architecture and Development Model. Earth Science_-Journal of China University of Geosciences, 34, 540-546. 
[8] Park, M.H., Kim, H.C. and Lee, S.J. (2013) Implementation Results and Service Examples of GPS-Tag for Indoor LBS and Message Service. Proceedings of 15th International Conference on Advanced Communication Technology (ICACT), Chiang Mai, 27-30 January 2013, 367-370.

[9] Deng, J., Zheng, X.Q. and Lv, L. (2011) A Method for Service Oriented WebGIS Construction by Integrating OpenSource GIS. 19th International Conference on Geoinformatics, Shanghai, 24-26 June 2011, 1-5

[10] Garbin, D.A. and Fisher J.L. (2010) Open Source for Enterprise Geographic Information Systems. IT Professional, 12, 38-45. 
Scientific Research Publishing (SCIRP) is one of the largest Open Access journal publishers. It is currently publishing more than 200 open access, online, peer-reviewed journals covering a wide range of academic disciplines. SCIRP serves the worldwide academic communities and contributes to the progress and application of science with its publication.

Other selected journals from SCIRP are listed as below. Submit your manuscript to us via either submit@scirp.org or Online Submission Portal.
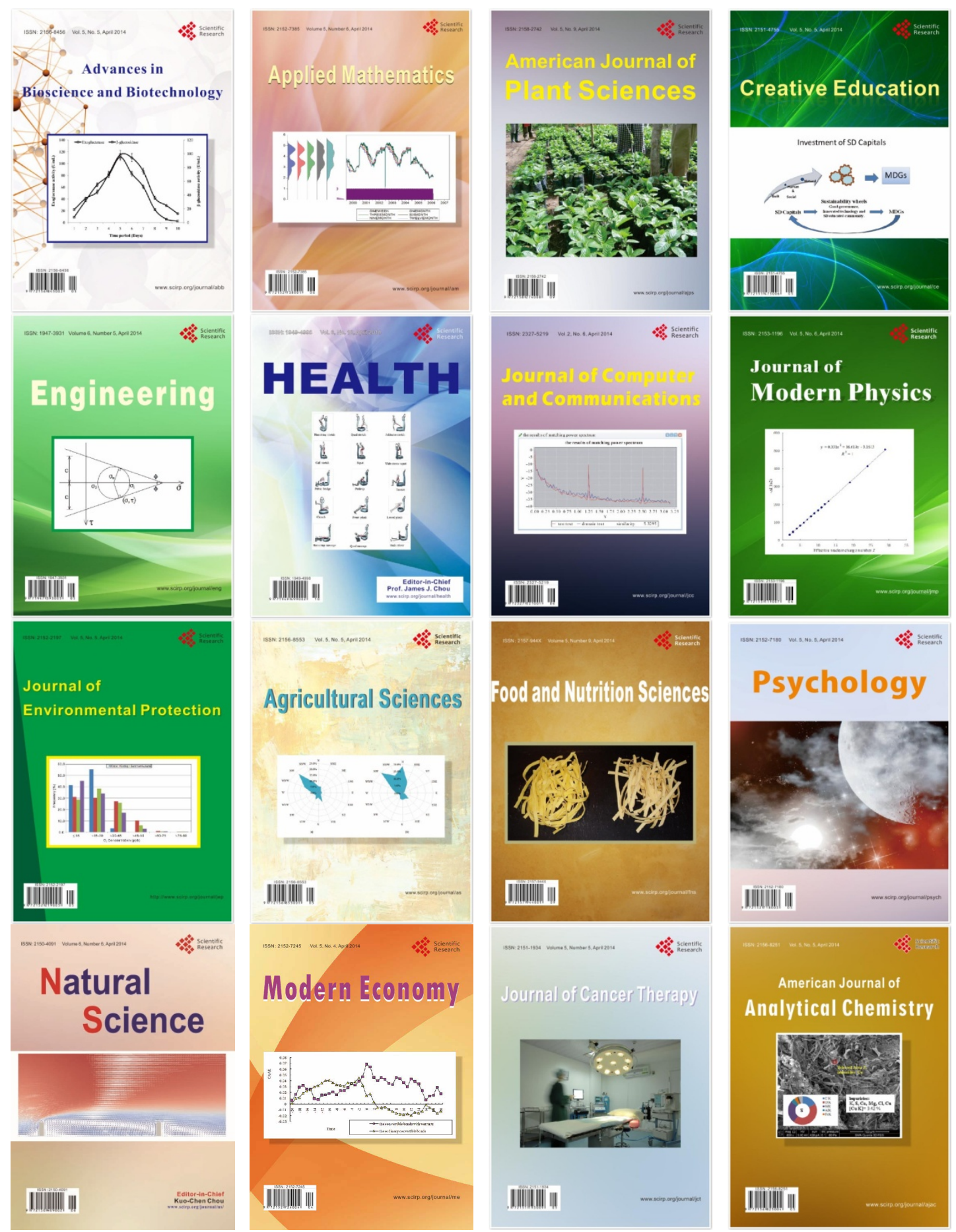